\title{
Mode-locking in vertical external-cavity surface-emitting lasers with type-II quantum-well configurations
}

Cite as: Appl. Phys. Lett. 114, 252102 (2019); https://doi.org/10.1063/1.5098903

Submitted: 04 April 2019 . Accepted: 13 June 2019 . Published Online: 25 June 2019

I. Kilen (D), S. W. Koch (D), J. Hader (D), and J. V. Moloney
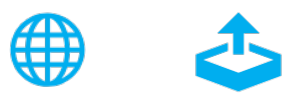

\section{ARTICLES YOU MAY BE INTERESTED IN}

Design guidelines for on-chip unstable resonator cavity to suppress filamentation in GaSbbased diode lasers

Journal of Applied Physics 125, 243101 (2019); https://doi.org/10.1063/1.5100268

Highly oriented graphite produced by femtosecond laser on diamond

Applied Physics Letters 114, 251903 (2019); https://doi.org/10.1063/1.5097986

Effect of exciton transfer on recombination dynamics in vertically nonuniform GaAsSb epilayers

Applied Physics Letters 114, 252101 (2019); https://doi.org/10.1063/1.5105343

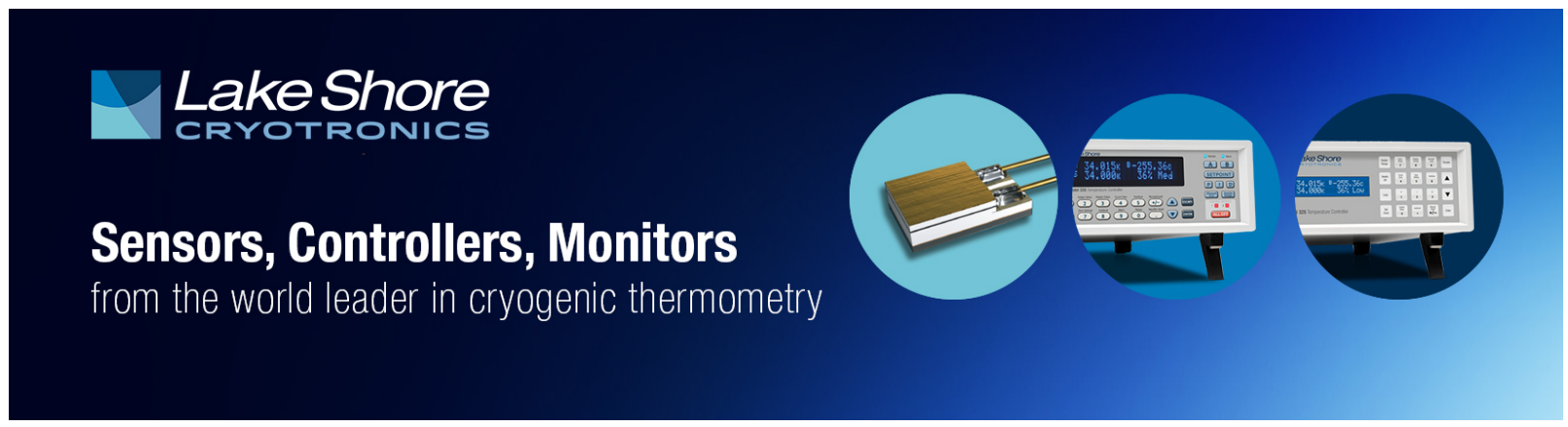




\title{
Mode-locking in vertical external-cavity surface-emitting lasers with type-II quantum-well configurations
}

Cite as: Appl. Phys. Lett. 114, 252102 (2019); doi: 10.1063/1.5098903

Submitted: 4 April 2019 - Accepted: 13 June 2019 .

Published Online: 25 June 2019

I. Kilen, ${ }^{1, a)}$ (D) S. W. Koch, ${ }^{1,2}$ (D) J. Hader, ${ }^{1,3}$ (D) and J. V. Moloney ${ }^{1,3,4}$

\author{
AFFILIATIONS \\ ${ }^{7}$ College of Optical Sciences, University of Arizona, 1630 East University Boulevard, Tucson, Arizona 85721, USA \\ ${ }^{2}$ Department of Physics and Material Sciences Center, Philipps-Universität Marburg, Renthof 5, 35032 Marburg, Germany \\ ${ }^{3}$ Nonlinear Control Strategies Inc., Tucson, Arizona 85704, USA \\ ${ }^{4}$ Department of Mathematics, University of Arizona, 617 N. Santa Rita Ave., Tucson, Arizona 85721, USA \\ a)ikilen@optics.arizona.edu
}

\begin{abstract}
A microscopic study of mode-locked pulse generation is presented for vertical external-cavity surface-emitting lasers utilizing type-II quantum well configurations. The coupled Maxwell semiconductor Bloch equations are solved numerically where the type-II carrier replenishment is modeled via suitably chosen reservoirs. Conditions for stable mode-locked pulses are identified allowing for pulses in the 100 fs range. Design strategies for type-II configurations are proposed that avoid potentially unstable pulse dynamics.
\end{abstract}

Published under license by AIP Publishing. https://doi.org/10.1063/1.5098903

Vertical external-cavity surface-emitting lasers (VECSELs) are highly versatile, relatively low-cost semiconductor disk lasers capable of continuous wave (CW) and mode-locked operation. ${ }^{1-4}$ Until now, most systems are based on quantum well (QW) heterostructures with type-I band alignment, ${ }^{5}$ i.e., configurations where the optical transitions involve electrons and holes in the same QW. Here, the highest peak power for multimode $\mathrm{CW}$ output has been reported as $106 \mathrm{~W}$, and single mode-operation has yielded $23 \mathrm{~W}$ with a distributed Bragg reflector $(\mathrm{DBR})^{8}$ and $16 \mathrm{~W}$ without a DBR. ${ }^{9}$ In mode-locked operation, pulses with temporal durations of about 100 fs (Refs. 10 and 11) and below ${ }^{12}$ have been realized.

In modern heterostructure growth, it is also possible to realize systems with a type-II band alignment, where the energetically lowest conduction band minima and the highest valence band maxima are in different QWs. ${ }^{5}$ Despite the fact that the optical transitions are spatially indirect in this case, rather large optical gain can be realized rivaling that of traditional type-I systems. ${ }^{13}$ Due to the added flexibility to separately optimize the two QW configurations involved in the type-II transition, one can realize optical transitions in a wider frequency range than possible with type-I systems. Moreover, the so-called type-II W-configurations have been designed for long-wavelength operation with the goal of reducing the intrinsic Auger losses and simultaneously maintaining high spectral amplification. ${ }^{14,15}$
Recently, W-well based VECSELs for operation in the $1200 \mathrm{~nm}$ wavelength regime have been realized and shown to reach $4 \mathrm{~W}$ of multimode and $0.35 \mathrm{~mW}$ of single-mode $\mathrm{CW}$ output power. ${ }^{16,17}$ In pulsed operation, electrical injection pumped edge-emitting gain chips based on type-II QW design have produced pulses with $1.4 \mathrm{~W}$ output power per facet. ${ }^{18}$ Not only has it been demonstrated that type-II QWs are able to reach new wavelength ranges but the studies of the systems in the $1.3 \mu \mathrm{m}$ region also showed that the type-II QW configuration tends to be less sensitive to changes in temperature. ${ }^{19}$

On the basis of these promising results, in particular, in view of the relatively wide flexibility to choose the optical transition frequency, it is interesting to investigate to which degree type-II W-laser configurations can be used for mode-locking and ultrashort pulse generation. For optical pumping into energetically high lying barrier states, it is very likely that the resulting, spatially separated densities of electrons and holes in the active lasing transition are somewhat different due to their intrinsically different relaxation processes. In mode-locked operation, the propagating intracavity pulse is amplified in the gain chip QWs and partially absorbed by the semiconductor saturable absorber mirror (SESAM). In order to obtain stable mode-locked pulses, it is mandatory that both the gain chip and the SESAM recover to the same state during every round trip. 
In comparison to type-I systems, the type-II QWs exhibit a somewhat different carrier recovery dynamics such that it is not immediately clear if one can match the gain chip and absorber dynamics allowing for mode-locking of the devices. Recent experimental measurements show that type-II QW gain recovery is slower than that of type-I QWs, ${ }^{20}$ which could adversely influence the pulse energy and mode-locking dynamics. Since the amount of amplification/absorption is nonlinearly dependent on the instantaneous QW carrier state, one has to systematically account for the microscopic QW carrier dynamics and couple this back to the pulse propagation in order to quantitatively model the mode-locking dynamics in type-II QWs.

In this paper, we investigate the potential for stable mode-locked VECSELs based on type-II QWs. For this purpose, we numerically solve Maxwell's equation for pulse propagation through the VECSEL coupled to the semiconductor Bloch equations (SBEs) that describe the macroscopic polarization of each QW. To keep the approach as simple as possible, we model the microscopic QW polarization dephasing and the carrier scattering with effective rates where the polarization relaxes to zero and the carriers scatter toward an instantaneous quasi-Fermi distribution. ${ }^{21,22}$ In the past, we used this scheme to analyze mode-locking in VECSELs with type-I QWs and successfully compared the outcome to experimental results ${ }^{12}$ such that, with the appropriate modification introduced here, this microscopically based model should be well suited for testing the stability of modelocking in type-II QWs.

We consider the generic structure configuration shown in Fig. 1. Here, the optical pump is absorbed in the GaAs barrier material and generates carriers which subsequently relax from the spatially delocalized initial states into the energetically lower confined QW states via carrier-carrier and carrier-phonon scattering. This carrier capture process has been modeled microscopically with good agreement to experimental observations. ${ }^{23}$ During and after the relaxation into the lower energy states, the carriers eventually equilibrate energetically close to the lasing transition.

Since the detailed, fully microscopic many-body calculation of the high energy carriers relaxing into the lower lasing states is computationally expensive, we introduce a simplified treatment that still allows us to capture the main features of a type-II W-laser configuration. The

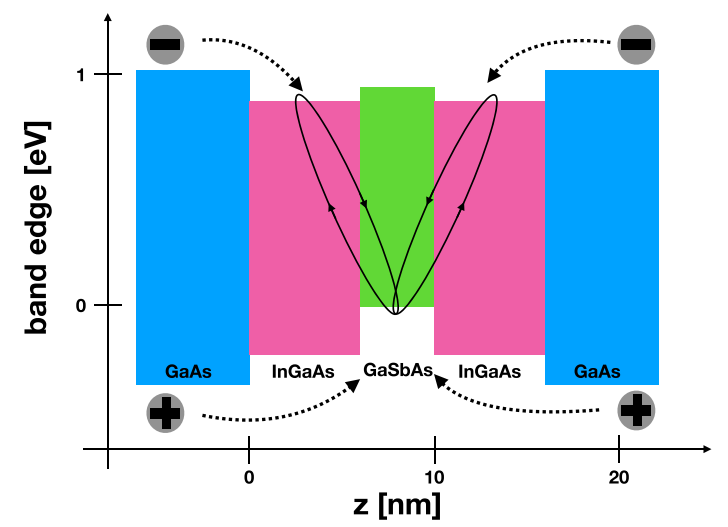

FIG. 1. A schematic of the spatial design of the type-II QW potentials with GaAs (blue), InGaAs (magenta), and GaSbAs (green) material layers. The solid arrows indicate the lasing transition simulated using the SBE. constant background of carriers, available to the QWs from barrier pump absorption, is modeled as a static Fermi distribution. These background carriers scatter into a QW high-energy reservoir state which will dynamically release carriers into the lower-energy lasing transition. Due to the different well configurations for electrons and holes in a type-II QW, the rate of transfer of carriers from the reservoir to the lasing transition may differ for electrons and holes, which in general will result in unequal densities in the active transition.

In our approach, the relaxation of carriers from the constant, pump induced background distributions, $\mathrm{F}_{\mathrm{k}}^{(\mathrm{e} / \mathrm{h})}$, to the $\mathrm{QW}$ reservoir is modeled using a scattering time $\tau_{\text {scatt }}$. The carriers from the reservoir are assumed to scatter into the lasing transition with a characteristic electron (hole) time $\tau_{e}\left(\tau_{h}\right)$. This relaxation is modeled using $-\left(\mathrm{n}_{\mathrm{k}}^{(\mathrm{e} / \mathrm{h})}\right.$ $\left.-\mathrm{n}_{\mathrm{k}}^{(\mathrm{e} / \mathrm{h}) \text {,res }}\right) / \tau_{(\mathrm{e} / \mathrm{h})}$. Figure 2 contains a schematic of this relaxation model. Following this, the reservoir electrons relax according to $\mathrm{d} / \mathrm{dt}_{\mathrm{k}}^{\mathrm{e}, \text { res }}(t)=-\left(\mathrm{n}_{\mathrm{k}}^{\mathrm{e}, \text { res }}-\mathrm{F}_{\mathrm{k}}^{\mathrm{e}}\right) / \tau_{\text {scatt }}+\left(\mathrm{n}_{\mathrm{k}}^{\mathrm{e}}-\mathrm{n}_{\mathrm{k}}^{\mathrm{e}, \text {,res }}\right) / \tau_{\mathrm{e}}$, with the corresponding equation for the holes.

With regard to the numerical values for the various relaxation times, we note that in experimentally realized heterostructures, the capture times depend on the details of the entire system which define

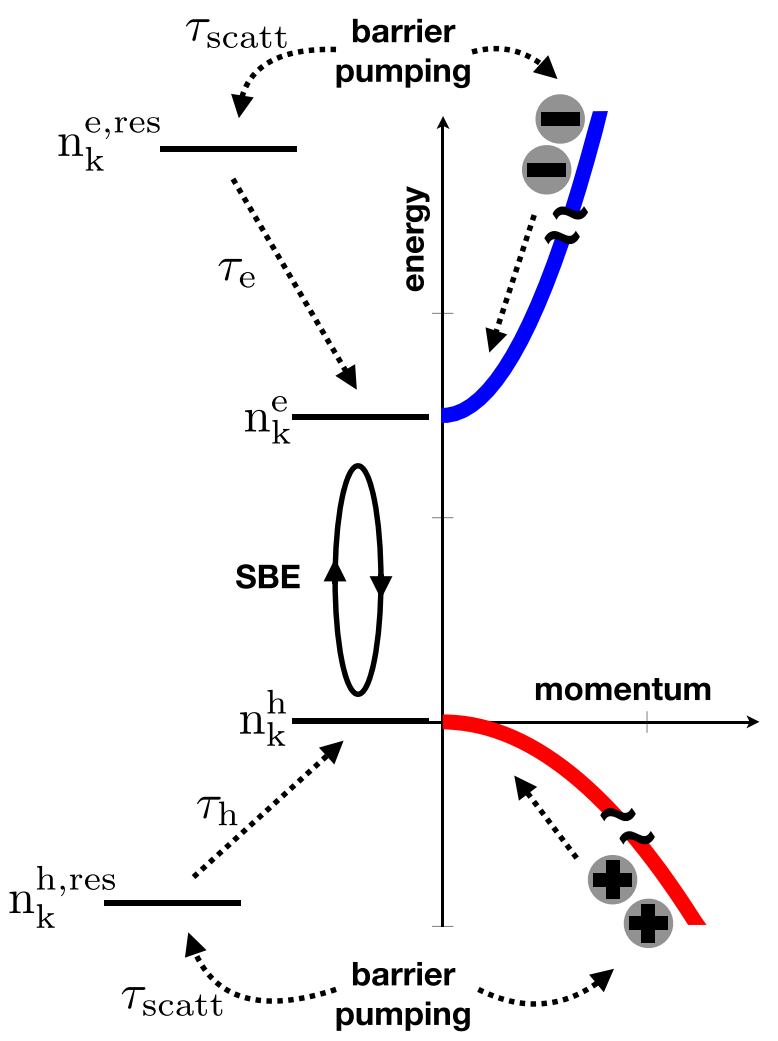

FIG. 2. A diagram showing the relaxation of barrier pumped carriers in a type-II QW and the important model parameters: the momentum resolved high energy reservoir carriers, $n_{k}^{(e / h) \text {,res }}$, that scatter to lower energy states and replenish the active lasing carriers, $n_{k}^{(e / h)}$, on a time scale $\tau_{(e / h)}$. Barrier pumped carriers relax into the reservoir at a time scale, $\tau_{\text {scatt. }}$. The dashed arrows indicate transitions that involve carrier scattering, and the solid arrows denote the lasing transition simulated using the SBE. 
the multitude of energy bands that characterize carriers in continuum (barrier states) and captured configurations in the QW. For barrier pumping, the microscopic mechanism of the capture process is a combination of Coulomb carrier-carrier and carrier-phonon scattering. The resulting capture rates will thus depend on the precise multiband configuration as well as on the energy and intensity of the pump. Using our fully microscopic analysis for a characteristic configuration (see the supplementary material), we find time scales on the order of ten picoseconds. Since the goal of this paper is a feasibility study for mode-locking in type-II W-laser configurations, we thus perform studies for a range of realistic capture rates in the range of 5 ps to 40 ps and identify their influence on the emitted pulse properties.

Before we proceed to a presentation of our numerical results, we want to note that one feature of our pump model is that the replenishment of the carrier density in the active lasing transition is limited by the reservoir carrier density level. During lasing operation, carriers are scattered into the reservoir on the time scale of $\tau_{\text {scatt }}$ and lost to the lasing transition on the time scale of $\tau_{(\mathrm{e} / \mathrm{h})}$. On this basis, the resulting dynamic interplay between carriers in the active lasing transition and reservoir produces two effective time scales for the carrier density recovery of the active lasing transition, $\mathrm{T}_{ \pm}^{(\mathrm{e} / \mathrm{h})}$, which are nonlinear combinations of the scattering times, $\tau_{\mathrm{e}}, \tau_{\mathrm{h}}$, and $\tau_{\text {scatt. }}$. The details on the derivation and analytical expression for these effective time scales in the absence of an electric field are found in the supplementary material. In particular, we identify a time scale for the slow recovery of carriers, $T_{-}^{(e / h)} \geq 2 \tau_{\text {scatt }}$, and this inequality becomes larger for increasing $\tau_{(\mathrm{e} / \mathrm{h})}$. The fast carrier recovery time satisfies, $0<\mathrm{T}_{+}^{(\mathrm{e} / \mathrm{h})}<\tau_{\text {scatt }}$, and approaches zero for shorter scattering times, $\tau_{(\mathrm{e} / \mathrm{h})}$. Note that, as a consequence of the two-stage relaxation model presented above, type-II QWs will have a slower gain recovery rate than type-I QWs using only $\tau_{\text {scatt }}$ as a characteristic time scale for carrier recovery.

To illustrate the generic similarities and differences, we analyze the influence of the type-II QW carrier relaxation on mode-locked pulses via a comparison to the results for the same VECSEL setup with type-I QWs. ${ }^{24}$ We choose a configuration where the gain chip consists of a DBR with 30 pairs of AlGaAs, an active region containing the QWs in a resonant periodic gain arrangement, an InGaP cap layer, and a dispersion compensating coating from $\mathrm{Ta}_{2} \mathrm{O}_{5} / \mathrm{SiO}_{2}$. In this design, 10 QWs are arranged on the antinodes of a standing wave from the DBR centered at $1030 \mathrm{~nm}$. The external cavity is configured with a simple SESAM on top of a $1 \%$ output coupling mirror separated from the gain chip by an air gap that results in a pulse round trip time of 21 ps. For the numerical investigations presented below, we use $\tau_{\text {scatt }}=30 \mathrm{ps}$, a lattice temperature of $300 \mathrm{~K}$, and a constant background density of $1.82 \times 10^{12} \mathrm{~cm}^{-2}$. As a comparison, the same VECSEL cavity with type-I QWs relaxing to the same background Fermi distribution with a single characteristic time scale, $\tau_{\text {scatt }}$ produced a mode-locked pulse with a full width at half maximum (FWHM) of $102 \mathrm{fs}$ and an output peak intensity of $0.16 \mathrm{MW} / \mathrm{cm}^{2}$.

Figure 3(a) shows an overview of stable mode-locked pulse peak intensities and temporal FWHM, produced with type-II QWs, for multiple different electron and hole scattering times. Here, we have sampled the hole scattering times according to $\tau_{\mathrm{h}}=\tau_{\mathrm{e}}+\tau_{\mathrm{d}}$, where $\tau_{\mathrm{d}}$ $=0 \mathrm{ps}$ (circles), $20 \mathrm{ps}$ (triangles), and $40 \mathrm{ps}$ (squares). For all values of $\tau_{\mathrm{d}}$ examined here, the pulse peak intensity increases by a factor of about 3 and the FWHM decreases by about $66 \%$ when the electron scattering time, $\tau_{\mathrm{e}}$, is reduced from 40 ps to 5 ps. On the other hand,
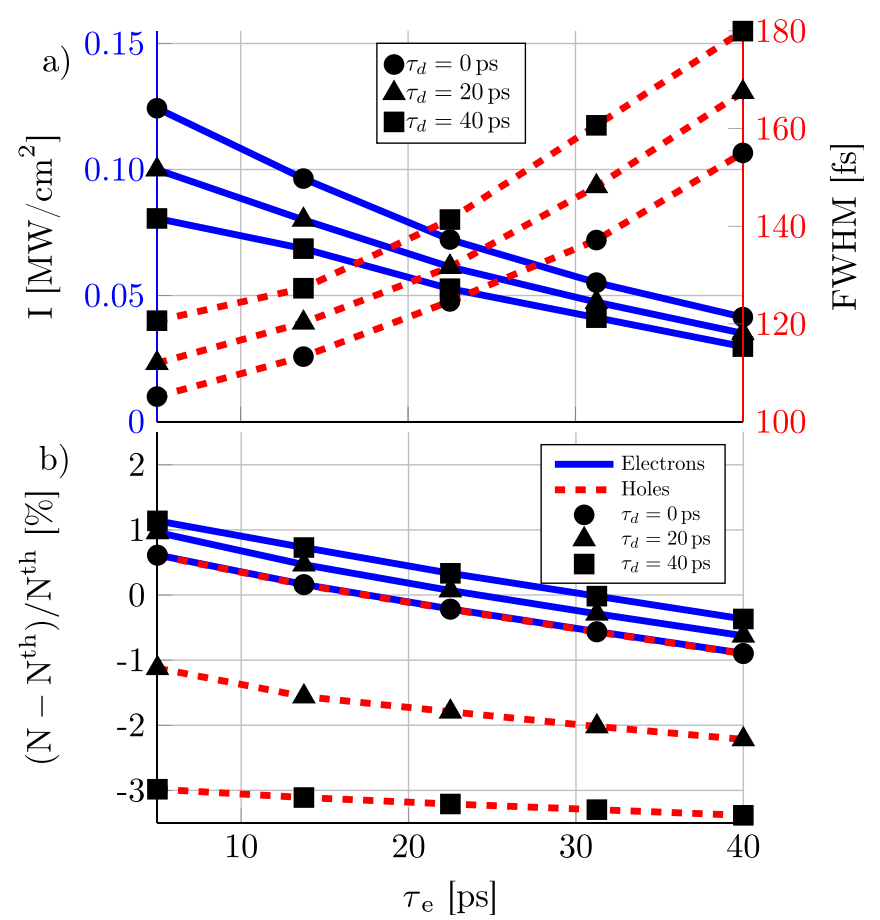

FIG. 3. A summary of mode-locked pulses from type-II QWs for electron scattering times, $\tau_{\mathrm{e}}$, with select hole rates given by $\tau_{\mathrm{h}}=\tau_{\mathrm{e}}+\tau_{\mathrm{d}}$, for $\tau_{\mathrm{d}}=0$ ps (circles), 20 ps (triangles), and 40 ps (squares). (a) The resulting mode-locked output peak intensity (blue) and FWHM (red). (b) The maximal QW carrier density during a round trip of mode-locked operation relative to the threshold density $\mathrm{N}^{\text {th }}$.

increasing the hole scattering time by $\tau_{\mathrm{d}}$ with a fixed $\tau_{\mathrm{e}}$ results in a $28 \%-35 \%$ decrease in pulse peak intensity and a $15 \%-16 \%$ increase in pulse FWHM. As expected, a lower scattering time results in faster carrier replenishing and more carriers in the lasing transition, thus supporting a higher pulse peak intensity. In contrast, a longer scattering time will reduce the QW gain and result in a higher pulse temporal duration. Overall, the pulse fluence decreases for longer carrier relaxation times because the intensity drops faster than the widening of the pulse. For the range of $\tau_{\mathrm{e}}$ examined here, the fluence drops by about a factor of 2 for a fixed $\tau_{\mathrm{d}}$ and, for a fixed $\tau_{\mathrm{e}}$, decreases by about $17 \%-20 \%$ with varying $\tau_{\mathrm{d}}$.

In Fig. 3(b), we have recorded the QW carrier density immediately before a mode-locked pulse extracts carriers. The densities are relative to the threshold density, $\mathrm{N}^{\text {th }} \approx 1.739 \times 10^{16} \mathrm{~m}^{-2}$, where the spectral amplification of the gain chip is equal to the cavity loss when the gain chip QW carriers are assumed to be in equilibrium at the background pump density. There is a $0.4 \%-1.6 \%$ increase in carrier density for fixed $\tau_{\mathrm{d}}$ when reducing the electron scattering time over this plot range. For fixed $\tau_{\mathrm{e}}$, the increased time difference, $\tau_{\mathrm{d}}$, results in about a $0.5 \%$ increase in electron density and a $2.5 \%-3.6 \%$ decrease in hole density. We expected the reduced hole density because $\tau_{\mathrm{h}}$ is increasing, which results in a slower transfer of carriers from the reservoir into the lasing transition. It is somewhat unexpected that the electron density slightly increases. This happens in order to compensate for the reduced hole density. The latter change is minimal, and the average density decreases by about $1 \%-2.5 \%$. 
Figure 3(b) shows that for longer electron scattering times, the nonequilibrium carrier density goes slightly below the threshold density, $\mathrm{N}^{\text {th }}$, for both electrons and holes. The threshold density is common in characterization of lasers and is significant because spontaneous emission cannot grow below this density. However, mode-locked pulses can operate stably below the threshold density ${ }^{25}$ because nonequilibrium pulse amplification is obviously different from CW operation and $\mathrm{N}^{\text {th }}$ is computed, while QW carriers are in equilibrium Fermi distributions. In our simulations, the pulse is first established using the background pump density of $1.82 \times 10^{16} \mathrm{~m}^{-2}$, which provides net amplification inside the VECSEL.

In Fig. 3, we see that the mode-locked pulse peak intensity depends critically on $\tau_{(\mathrm{e} / \mathrm{h})}$. The mode-locked pulses from type-II QWs are both longer and have lower peak intensities than the corresponding type-I QWs. A longer electron and hole relaxation time will in general result in lower mode-locked pulse energy. We have not attempted to optimize the type-II QWs for ultrashort pulse generation or high peak intensity at this time. However, the results show that a shorter electron/hole relaxation time will result in more carriers in the lasing transition, which will give shorter FWHM and higher peak intensity.

When comparing Figs. 3(a) and 3(b) over the given range of electron scattering times, we observe that the pulse fluence decreases by a factor of 2 , while the total carrier density only decreases by $1 \%-2.5 \%$. It is not surprising that very similar carrier densities can produce very different mode-locked pulses because the total carrier density is not directly available to the pulse itself. Instead, the pulse can only extract energy from a subset of carriers in each QW, namely, the inverted carriers. ${ }^{21}$ For type-II QWs, the principal difference is that the electron and hole occupation numbers will relax differently based on their respective relaxation times and result in unequal electron and hole densities. However, the pulse still interacts with the QW inversion, $n_{k}^{\mathrm{e}}+n_{k}^{\mathrm{h}}-1$, that depends on the momentum resolved carrier occupation numbers.

Our detailed analysis indicates that the reservoir relaxation and the associated electron and hole relaxation times can become a source for instabilities in the mode-locking dynamics. To see this, we first consider a typical example with a stable mode-locked pulse. Figure 4 shows an example of the total density of reservoir and active lasing carriers during stable mode-locked operation. Note that the drop of

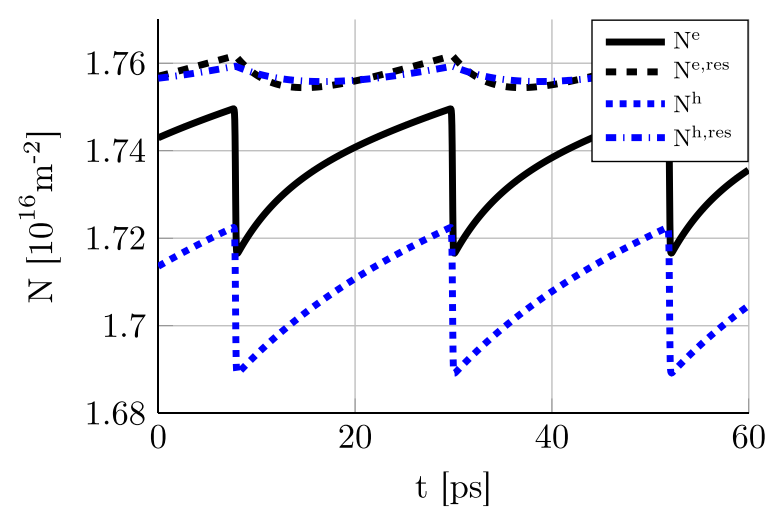

FIG. 4. Total density of electrons and holes in the reservoir and in the active transition during stable mode-locked operation. In this example, $\tau_{e}=10 \mathrm{ps}$ and $\tau_{\mathrm{h}}=25 \mathrm{ps}$. carriers is due to the propagating mode-locked pulse extracting carriers each round trip. A stable mode-locked pulse appears when the reservoir maintains a sufficient reserve of carriers and the lasing transition has enough carriers to support the propagating mode-locked pulse. This case was examined in detail in Fig. 3. Another limit to consider is when the electron/hole relaxation times become very long compared to the reservoir relaxation time. In this limit, there are not enough carriers in the lasing transition to produce a mode-locked pulse with sufficient energy to bleach the SESAM, which will prevent the formation of a stable mode-locked pulse.

A true nonequilibrium pulse instability can appear when one or both of the electron/hole relaxation times are sufficiently short. In this case, too many carriers relax into the lasing transition during interaction with the single mode-locked pulse, and the unsaturated carriers attempt to produce a second pulse immediately following the first. This case is similar to a previously studied situation where multiple cavity pulses were created because of too high QW carrier density. ${ }^{21}$ The main difference is that the current unstable pulse form does not break apart into multiple pulses. The tail behind the leading pulse will, over thousands of round-trips, grow into a secondary pulse that extracts energy from a spectral region near the carrier reservoir of the original pulse. The system attempts to accommodate both carrier reservoirs but is unable to find a balance that supports two pulses. The leading pulse is suppressed in favor of the secondary pulse, and this process repeats by creating a tail behind the new pulse. This situation can be avoided, and stable mode-locking can be established by reducing the density of the background barrier distribution, $\mathrm{F}_{\mathrm{k}}^{(\mathrm{e} / \mathrm{h})}$, i.e., by reducing the pump. The supplementary material contains more details on this instability.

Altogether, we identify three stability regimes for mode-locked pulses that can be characterized by looking at the reservoir carriers. The mode-locked pulse is stable when the reservoir has a sufficient carrier density to replenish the active lasing transition, i.e., the electron/hole relaxation times are not too short and thus allow for a balance between the reservoir and lasing transition carriers. Unstable lasing occurs when the reservoir carriers relax too quickly into the lasing transition during interaction with the pulse. This fast recovery can produce unsaturated carriers that in turn can cause an additional pulse to appear in a time window after the original cavity pulse. Finally, too long electron/hole relaxation times result in no mode-locked pulse in the cavity.

In summary, we presented numerical results analyzing the feasibility to generate short pulse mode-locking in type-II QW W-laser configurations. We predict stable mode-locked pulses with temporal durations slightly above 100 fs and peak intensities around $0.1 \mathrm{MW} /$ $\mathrm{cm}^{2}$. We find stable mode-locked pulses with somewhat lower peak intensities and longer FWHM than the corresponding VECSEL with type-I QWs. Whereas the pulse details depend on the exact system parameters, the overall mode-locked pulse behavior is rather robust against small changes in the exact carrier capture dynamics. We derived analytical expressions that should help with the design of typeII QW based gain chips, where the type-II QW configuration allows for increased flexibility to choose the central pulse emission wavelength.

See the supplementary material for the derivation of the carrier recovery time scales, $\mathrm{T}_{ \pm}$, details on the unstable mode-locked pulse, 
and an example many-body calculation of carriers scattering into the type-II QW.

This material is based upon the work supported by the Air Force Office of Scientific Research under Award No. FA9550-17-10246. The Marburg research was supported by the Deutsche Forschungsgemeinschaft via the Sonderforschungsbereich 1083. We thank Wolfgang Stolz for insightful discussions.

\section{REFERENCES}

${ }^{1}$ U. Keller and A. C. Tropper, Phys. Rep. 429, 67 (2006).

${ }^{2}$ A. C. Tropper, A. H. Quarterman, and K. G. Wilcox, Adv. Semicond. Lasers 86, 269 (2012).

${ }^{3}$ B. W. Tilma, M. Mangold, C. A. Zaugg, S. M. Link, D. Waldburger, A. Klenner, A. S. Mayer, E. Gini, M. Golling, and U. Keller, Light: Sci. Appl. 4, e310 (2015).

${ }^{4}$ A. Rahimi-Iman, J. Opt. 18, 093003 (2016).

${ }^{5}$ H. Kroemer, Phys. Scr. 1996, T68.

${ }^{6}$ B. Heinen, T.-L. Wang, M. Sparenberg, A. Weber, B. Kunert, J. Hader, S. W. Koch, J. V. Moloney, M. Koch, and W. Stolz, Electron. Lett. 48, 516 (2012).

${ }^{7}$ T.-L. Wang, B. Heinen, J. Hader, C. Dineen, M. Sparenberg, A. Weber, B. Kunert, S. W. Koch, J. V. Moloney, M. Koch, and W. Stolz, Laser Photonics Rev. 6, L12 (2012).

${ }^{8}$ F. Zhang, B. Heinen, M. Wichmann, C. Möller, B. Kunert, A. Rahimi-Iman, W. Stolz, and M. Koch, Opt. Express 22, 12817 (2014).

${ }^{9}$ Z. Yang, D. Follman, A. Albrecht, P. Heu, N. Giannini, G. Cole, and M. SheikBahae, Electron, Lett. 54, 430 (2018).
${ }^{10}$ D. Waldburger, S. M. Link, M. Mangold, C. G. E. Alfieri, E. Gini, M. Golling, B. W. Tilma, and U. Keller, Optica 3, 844 (2016).

${ }^{11}$ P. Klopp, U. Griebner, M. Zorn, and M. Weyers, Appl. Phys. Lett. 98, 071103 (2011).

${ }^{12}$ A. Laurain, I. Kilen, J. Hader, A. Ruiz Perez, P. Ludewig, W. Stolz, S. Addamane, G. Balakrishnan, S. W. Koch, and J. V. Moloney, Appl. Phys. Lett. 113, 121113 (2018).

${ }^{13}$ C. Bückers, A. Thränhardt, S. W. Koch, M. Rattunde, N. Schulz, J. Wagner, J. Hader, and J. V. Moloney, Appl. Phys. Lett. 92, 071107 (2008).

${ }^{14}$ J. Meyer, C. Hoffman, F. Bartoli, and L. Ram-Mohan, Appl. Phys. Lett. 67, 757 (1995).

${ }^{15} \mathrm{G}$. Zegrya and A. Andreev, Semiconductor 2, 3 (1996).

${ }^{16}$ C. Möller, C. Fuchs, C. Berger, A. Ruiz Perez, M. Koch, J. Hader, J. V. Moloney, S. W. Koch, and W. Stolz, Appl. Phys. Lett. 108, 071102 (2016).

${ }^{17}$ C. Möller, F. Zhang, C. Fuchs, C. Berger, A. Rehn, A. R. Perez, A. RahimiIman, J. Hader, M. Koch, J. V. Moloney et al., Electron. Lett. 53, 93 (2017).

${ }^{18}$ C. Fuchs, C. Berger, C. Möller, M. Weseloh, S. Reinhard, J. Hader, J. V. Moloney, S. W. Koch, and W. Stolz, Electron. Lett. 52, 1875 (2016).

${ }^{19}$ C. Fuchs, A. Brüggemann, M. Weseloh, C. Berger, C. Möller, S. Reinhard, J. Hader, J. Moloney, A. Bäumner, S. Koch et al., Sci. Rep. 8, 1422 (2018).

${ }^{20}$ C. Lammers, M. Stein, C. Berger, C. Möller, C. Fuchs, A. Ruiz Perez, A. Rahimi-Iman, J. Hader, J. Moloney, W. Stolz et al., Appl. Phys. Lett. 109, 232107 (2016).

${ }^{21}$ I. Kilen, J. Hader, J. V. Moloney, and S. W. Koch, Optica 1, 192 (2014).

${ }^{22}$ I. Kilen, S. W. Koch, J. Hader, and J. V. Moloney, J. Opt. Soc. Am., B 33, 75 (2016).

${ }^{23}$ J. Hader, J. V. Moloney, and S. W. Koch, Appl. Phys. Lett. 85, 369 (2004).

${ }^{24}$ I. Kilen, S. W. Koch, J. Hader, and J. V. Moloney, Appl. Phys. Lett. 112, 262105 (2018).

${ }^{25}$ I. Kilen, S. W. Koch, J. Hader, and J. V. Moloney, Optica 4, 412 (2017). 